\title{
In Silico Screening of DNA Gyrase B Potent Flavonoids for the Treatment of Clostridium difficile Infection from PhytoHub Database
}

Kanika Verma ${ }^{1}$

https://orcid.org/0000-0002-9279-2759

\section{Panupong Mahalapbutr ${ }^{2}$}

https://orcid.org/0000-0003-4389-334X

Utid Suriya ${ }^{3}$

https://orcid.org/0000-0001-7742-8249

\section{Tuanjai Somboon ${ }^{1}$}

https://orcid.org/0000-0003-1372-2494
Thitinan Aiebchun ${ }^{1}$

https://orcid.org/0000-0001-8155-1602

\author{
Liyi Shi ${ }^{4}$ \\ https://orcid.org/0000-0003-1551-3768 \\ Phornphimon Maitarad 5 \\ https://orcid.org/0000-0003-0035-0070
}

Thanyada Rungrotmongkol ${ }^{1,6}$ *
https://orcid.org/0000-0002-7402-3235

${ }^{1}$ Chulalongkorn University, Faculty of Science, Department of Biochemistry, Biocatalyst and Environmental Biotechnology Research Unit, Bangkok, Thailand; ${ }^{2}$ Khon Kaen University, Faculty of Medicine, Department of Biochemistry, Khon Kaen, Thailand; ${ }^{3}$ Chulalongkorn University, Faculty of Science, Program in Biotechnology, Bangkok, Thailand; ${ }^{4}$ Shanghai University, College of Sciences, Department of Chemistry, Research Center of Nano Science and Technology, Shanghai , China; ${ }^{5}$ Shanghai University, Research Center of Nano Science and Technology, Baoshan district, Shanghai, People's Republic of China; ${ }^{6}$ Chulalongkorn University, Graduate School, Program in Bioinformatics and Computational Biology, Bangkok, Thailand.

Editor-in-Chief: Paulo Vitor Farago

Associate Editor: Jane Budel

Received: 2020.06.24; Accepted: 2021.02.17.

*Correspondence: thanyada.r@chula.ac.th; Tel +66-2218-5426 (R.T.).

\section{HIGHLIGHTS}

- Flavonoids potentially target GyrB.

- Screened flavonoids have good bioavailability and no toxicity.

- Flavonoids could preferentially interact with GyrB and some of them exhibit higher binding affinity towards GyrB than novobiocin.

\begin{abstract}
Clostridium difficile infection (CDI) is the most common hospital acquired diarrheal disease with its increasing incidence and mortality rate globally. DNA Gyrase B (GyrB) is a key component of DNA replication process across all bacterial genera; thus, this offers a potential target for the treatment of CDI. In the present study, several virtual screening approaches were employed to identify a novel $C$. difficile GyrB inhibitor. The 139 known metabolites were screened out from the 480 flavonoids in PhytoHub database. Molinspiration and PROTOX II servers were used to calculate the ADME properties and oral toxicity of the metabolites, whereas mutagenicity, tumorigenicity, irritant, and reproductive effect were predicted using DataWarrior program. The binding mode and the binding efficiency of the screened flavonoids against the GyrB were studied using
\end{abstract}


FlexX docking program. From virtual screening of 139 metabolites, we found 25 flavonoids with no mutagenicity, tumorigenicity, irritant, and reproductive effect. Docking study suggested that flavonoids 1030 ((-)-epicatechin 3'-O-sulfate), 1032 ((-)-epicatechin 4'-O-sulfate), 1049 (3'-O-methyl-(-)-epicatechin 4-Osulfate), 1051 (3'-O-methyl-(-)-epicatechin 7-O-sulfate), 1055 (4'-O-methyl-(-)-epicatechin 7-O-sulfate) and 1317 (quercetin sulfate) have significantly higher binding affinity than the known GyrB inhibitor novobiocin. The results from molecular dynamics simulation and free energy calculations based on solvated interaction energy suggested that (-)-epicatechin 3'-O-sulfate could be a potential drug candidate in the management of CDI.

Keywords: Clostridium difficile infection; Virtual screening; ADMET.

\section{INTRODUCTION}

Clostridium difficile, a gram-positive spore-forming anaerobic bacterium, is one of the leading pathogens that cause nosocomial infections [1]. Clostridium difficile infection (CDI) is the most common hospital acquired diarrheal disease with its increasing incidence and mortality rate globally. There are many reports regarding community acquired $C$. difficile infections (CA-CDI) that sprung in the U.S. between 1991 and 2005 [2, 3]. A CDI clinical symptom ranges from asymptomatic colonization to life-threatening colitis. CDI is caused by being in contact with $C$. difficile spores, receiving treatments like regular intake of antibiotics that could destroy the normal microflora of the intestinal tract, and the absence of well-being of the person such as old age, critical current diseases, decreased immune response, and damaged immunity [3]. Symptoms of CDI include watery diarrhea, fever, loss of appetite, nausea, and abdominal pain or tenderness. However, many bacterial development processes depend mainly on local unwinding of duplex DNA to transcribe its genetic information [4]. To determine consequent DNA supercoils, knots, and recombination intermediates, many microorganisms including bacteria have an advanced class of enzymes known as topoisomerase that relieves the adverse impacts of overwound and entangled chromosomes [5]. The most widely studied member of type IIA topoisomerase is DNA gyrase. DNA Gyrase B (GyrB, Figure 1), consists of the N-terminal ATPase domain, whereas the $\mathrm{C}$-terminal region includes Toprim domain and Tail based [6-8]. GyrB utilizes the energy of ATP hydrolysis to introduce negative supercoils into DNA. It is vital for all types of bacteria, due to its key role in the process of transcription and replication [6,8]. Inhibiting GyrB by small molecules has been an important mechanism in the treatment of bacterial infections. Many GyrB inhibitors have been reported with antibacterial activity such as novobiocin, coumermycin A1, clorobiocin, etc. which are categorized as aminocoumarin [7]. Among them, novobiocin is the most potent and widely used antibiotic to target GyrB [9-11]. It is also known as "golden era" of antibiotics as this was the first drug in the category of aminocoumarins [12] for the treatment of bacterial diseases. Novobiocin is a natural product which is isolated from the bacteria streptomyces [13]. It competitively inhibits GyrB at the ATPase domain of the bacterial DNA gyrase enzyme which is involved in the energy transduction [14]. Novobiocin binds to DNA gyrase, blocking its activity [7,11]. However, it is withdrawn from the market due to its side effects and efficacy issues [7]. In addition, novobiocin has been found to be ineffective and resistant $[11,15]$ towards many bacterial species such as Staphylococci $[7,16]$, facilitating their spread, which leads to bacterial infection. Therefore, there has been a keen interest in the exploration of potential plant-derived small molecules against GyrB for the treatment of $\mathrm{CDI}$ and to overcome the infection problem.

Flavonoids are well known category of phenolic compounds [17] and are widely found in various fruits and vegetables such as soy, honey, tea, nuts, and wines [18]. They have various medicinal properties such as antiviral, antimicrobial, antiallergic, and anti-inflammatory properties [18]. Notably, flavonoids have been used as dietary supplements or herbal medicines due to their nontoxic therapeutic effects [19]. Several flavonoid analogs have been reported to inhibit GyrB by interacting with the ATPase domain [20]. Moreover, quercetin, a widely known flavonoid, binds to the ATP-binding pocket of GyrB with a $K_{\mathrm{D}}$ value of $15 \mu \mathrm{M}$ and inhibits the ATPase activity of GyrB [21]. Recent studies also highlight that molecular dynamics simulation is one of the vital techniques these days and it has significant impact in understanding the dynamic behavior of the protein complex and the structural and functional elements changes at atomic level [22-25]. Computer aided simulations are also utilized in several areas of research such as identification and optimization of potent inhibitors to explore drug resistance mechanism and to determine protein-protein interactions [26,27]. In the present study, we aimed to replace the antibiotics which damage the normal flora of the intestine [2830] by introducing plant-derived compounds which can have potential health benefits and limited or no side effects. Thus, we screened flavonoids from the PhytoHub database [31] to identify a potential candidate 
against CDI. The obtained results could possibly provide further valuable insights into CDI and will help to gain better understanding of the role of GyrB as target in the treatment of CDI.

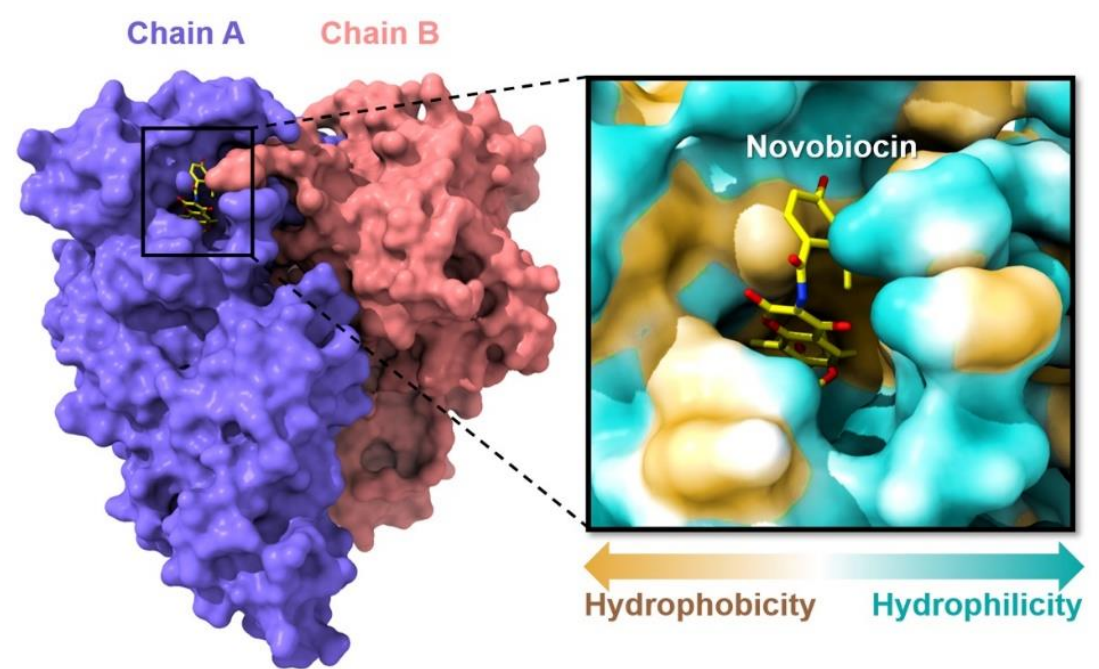

Figure 1. The 3D homology model of the ATPase domain of $C$. difficile GyrB protein, in which novobiocin in the ATPbinding pocket is shown in yellow stick. The coordinate of novobiocin was obtained from the PDB entry 1KIJ [32].

\section{MATERIAL AND METHODS}

\section{Homology modeling}

Homology modelling is a process where the three-dimensional structure of a protein is modelled using a known experimental structure of a homologous protein or its template [33]. The amino acid sequence of the protein was retrieved in FASTA format from Uniprot database (UniProtKB - Q18C89). The 3D structure of GyrB of C. difficile was modeled using SWISS-MODEL server [34,35] using Thermus thermophilus GyrB in complex with novobiocin (PDB ID: 1KIJ [32]) as template. Subsequently, the stereochemical quality of the 3D structure was evaluated using RAMPAGE: Ramachandran Plot Assessment, online version [36]. This program predicts the conformation of the polypeptide backbone via the phi/psi torsion angles from the amino acid sequence. If the determination of the structure is reliable, it is presumed that most pairs will be within the core or the favored regions of the plot. All ionizable amino acids ( $D, E, K, R$, and $H$ ) of the protein were protonated at $\mathrm{pH} 6.0$ using PROPKA 3.0 [37]. In addition, the AMBER ff14SB force field [38] was applied for the protein. In the present study, the protonated structure was used for further analysis. 


\section{Virtual screening}

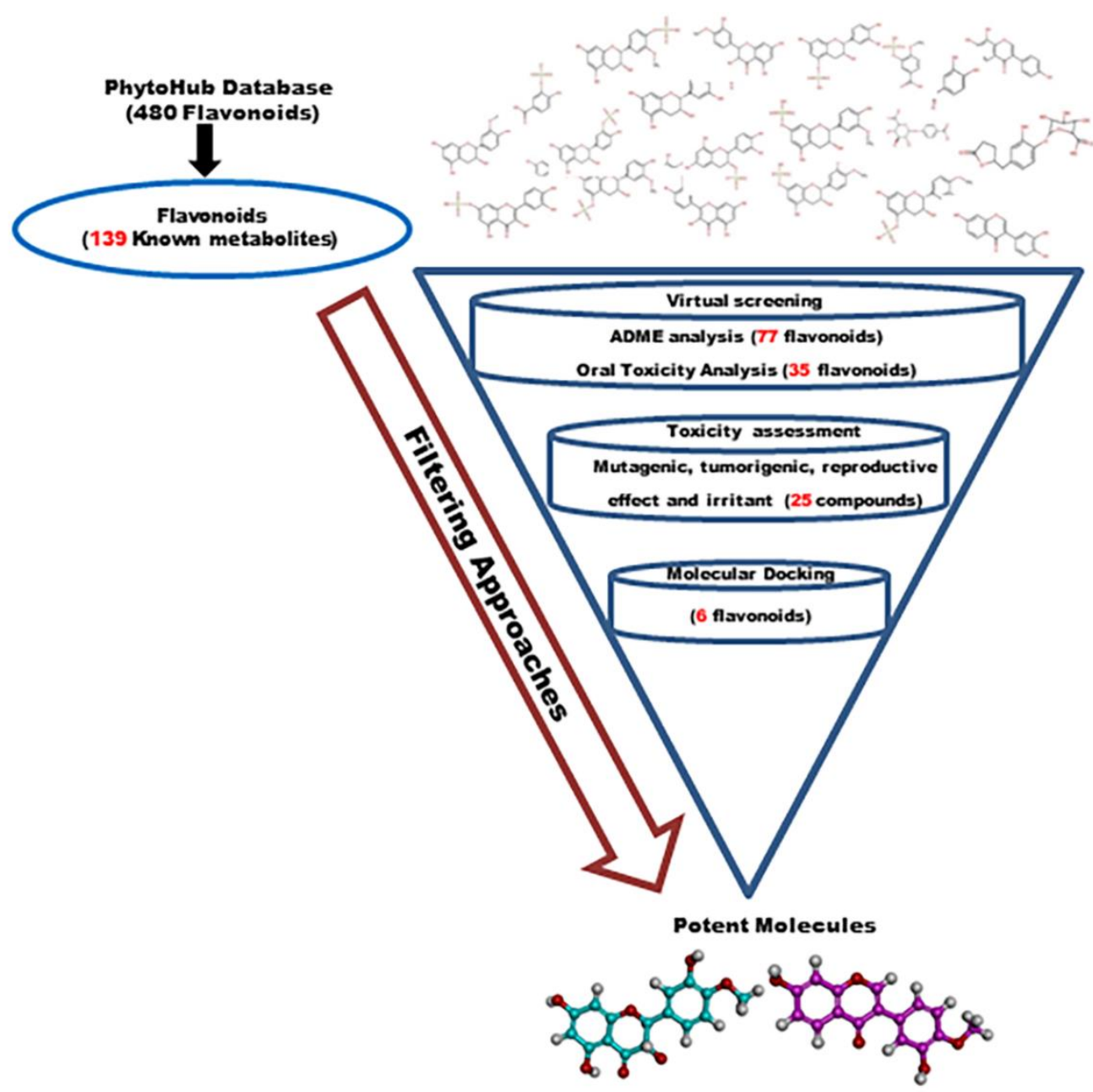

Figure 2. Diagrammatic representation of the work done in the present study.

\section{ADMET Analysis}

ADMET (Absorption, Distribution, Metabolism, Excretion and Toxicity) properties play a key role in the process of drug screening as it accounts for the failure of almost $60 \%$ of drugs in clinical phase trials. Thus, it is very important to carry out ADME analysis in the early phase of drug development. In order to eliminate the molecules with poor bioavailability, the various molecular descriptors and physiochemical properties were calculated for all 139 known metabolites of flavonoids from PhytoHub database and novobiocin used as reference drug by employing Molinspiration program (http://www.molinspiration.com). It calculates physicochemical parameters such as miLogP, molecular weight, TPSA (topological polar surface area) value, the number of hydrogen bond acceptor, hydrogen bond donor and rotatable bonds. These physiochemical properties are 9 together contributed to formulate Rule of Five (Lipinski Rule of Five) [44]. Subsequently, the oral toxicity analysis was performed using ProTox-II server [45]. The oral toxicity analysis is a very important part for the process of drug design and development. The ProTox calculation is based on 2D similarity to molecules exhibiting known $L_{50}$ values and on the recognition of chemical fragments that were overrepresented in compounds found to be toxic [46]. According to the Globally Harmonised System (GHS), toxicity is categorized into the six different classes based on $L D_{50}$ range [47] published by the Occupational Safety and Health Administration (OSHA) [48] (Supporting Information Table S2).

\section{Toxicity feature valuation}

The analysis for toxicity parameters were carried out by employing DataWarrior program for the screened molecules from ADMET analysis. The toxicity features (mutagenicity, tumorigenicity, irritant and reproductive effect) of the compounds were estimated based on the precompiled lists of fragments available using the 
algorithm that gives rise in the toxicity alerts in case that the compound structure is similar to that of the list of toxic fragments [48].

\section{Molecular docking}

The protonation state of each molecule was determined at $\mathrm{pH} 6$ using MarvinSketch implemented in ChemAxon software [49-51]. Protonation state has major role in the protein ligand binding. Also, the docking results depends on several factors such as protonation sites of protein and the ligand $[52,53]$. Then, the initial structure of ligands were generated using Gaussian program according to the standard protocols [54-56]. The optimized ligands were individually docked into the ATPase domain of GyrB with 100 docking runs using FlexX docking program [57]. FlexX docking is a best enrichment tool in structure-based drug design. It helps to generate precise poses of a ligand structure in target protein binding site. Moreover, it docks huge compound libraries by using ultra-high speed docking [58].The poses are scored and the conformer with the lowest docking score was considered to be the most probable binding confirmation of the ligand to the protein.

\section{System Preparation and MD simulations}

The antechamber and parmchk modules of AMBER16 were employed to generate the restrained ESP charges and missing parameters of all studied compounds. The bonded and non-bonded interaction parameters for the protein were treated with the AMBER ff14SB force field26 and generalized AMBER force field version 2 (GAFF2) [59], accordingly. The hydrogen atoms were added using the LEaP module implemented in AMBER16. In solvation, the TIP3P water model [60] was used in order to solvate the system with a minimum padding of $10.0 \AA$ between the protein surface and the solvation box edge, and the box dimensions were approximately set to $114 \times 97 \times 92 \AA^{3}$. To neutralize the overall charge of the molecular system, either sodium or chloride ions were randomly added. Minimization of the added hydrogen atoms and water molecules were carried out using 500 steps of steepest descent (SD) followed by 1500 steps of conjugated gradient (CG) methods prior to run the MD simulations, while the rest of the molecules were fixed. Subsequently, each protein-protein complex was minimized by means of SD (500 iterations) and CG (1500 iterations) with constrained solvent molecules. Lastly, the whole complex was fully minimized using the same procedure.

The studied systems were simulated under the periodic boundary condition with the isothermal-isobaric (NPT) scheme, as previously described [61-65]. In short, the electrostatic interactions were treated by the particle mesh Ewald summation method [66] and a cutoff distance for non-bonded interactions was set to 10 $\AA$. The SHAKE algorithm [67] was employed to constrain all covalently connected hydrogen atoms. Controlling the temperature and pressure were done by the Langevin thermostat [68] with a collision frequency of $2 \mathrm{ps}^{-1}$ and the Berendsen barostat [69] with a pressure relaxation time of $1 \mathrm{ps}$, respectively. In the heating step, temperature in each simulated system was gradually increased from 10 to $300 \mathrm{~K}$ set for 200 ps with a harmonic positional restraint of $30.0 \mathrm{kcal} / \mathrm{mol} \AA^{2}$ to the Ca atoms of protein. Subsequently, four steps of restrained MD simulations at $300 \mathrm{~K}$ were responsible for equilibrating the system of each complex, while harmonic restraints were set to $30,20,10$ and $5 \mathrm{kcal} / \mathrm{mol} \AA^{2}$ for overall 1300 ps and another 200 ps without any restraint. The entire system was then simulated under the NPT ensemble $(300 \mathrm{~K}, 1 \mathrm{~atm})$ until reaching $50 \mathrm{~ns}$. The MD production for all systems was set to $50 \mathrm{~ns}$ by increasing the time step in a 2-fs increment, while each MD trajectory was saved every 10 ps.

To evaluate the binding affinity, the total binding free energy ( $\left.\Delta G_{\text {binding }}\right)$ of each complex was calculated based upon solvated interaction energy (SIE) [70] approach. The general terms to estimate $\Delta \mathrm{G}_{\text {bind }}$ of a protein with a ligand in a solvent system are expressed in Equation 1 below.

$$
\Delta \mathrm{G}_{\text {bind }}(\mathrm{SIE})=\text { alpha }\left(\Delta \mathrm{E}_{\mathrm{vdW}}+\Delta \mathrm{E}_{\text {ele }}+\mathrm{RF}+\text { cavity }\right)+\text { constant }
$$

For this purpose, the MD trajectories were extracted for 100 snapshots from the last $10 \mathrm{~ns}$ (40-50 ns) of the MD production in order to calculate each energy component, which collectively represents the $\Delta \mathrm{G}_{\text {binding }}$ of each simulated protein-ligand complex. All data used to represent protein-ligand interactions and the binding free energies calculations were done by using the CPPTRAJ [71] and MMPBSA.py [72] modules of AMBER16, respectively. 


\section{RESULTS}

\section{Generation of 3D model and validation}

The quality of the modeled structure of ATPase domain of $C$. difficile GyrB protein generated by Phyre2 was evaluated using PROCHECK software [73]. The Ramachandran plot is displayed in Figure 3, and the results showed that the psi and phi angles are mainly presented in favored and allowed regions (99\%), indicating a reliable model.

Virtual Screening (VS) is a computer-based technique which helps to identify new potent molecules that binds to the target protein efficiently [39]. This approach is becoming popular in the pharma industry for the identification of lead molecule [40]. It helps to screen against a target protein specificaly, to a limited number of compounds that inhibit a maximum chance to a lead to be a drug candidate [40]. VS approach is mainly employed for screening large data set of compounds in a short time period and at low cost [41]. There are two types of VS approaches ligand (pharmacophore based) or structure-based (docking) VS techniques which are broadly used in high-throughput screening [42]. However, the widely used VS technique is molecular docking, where a drug candidate is placed into the active site of the target protein virtually and its binding affinity is calculated based on binding energy scores [43]. Figure 2 shows the flow of the VS work carried out in the present study.

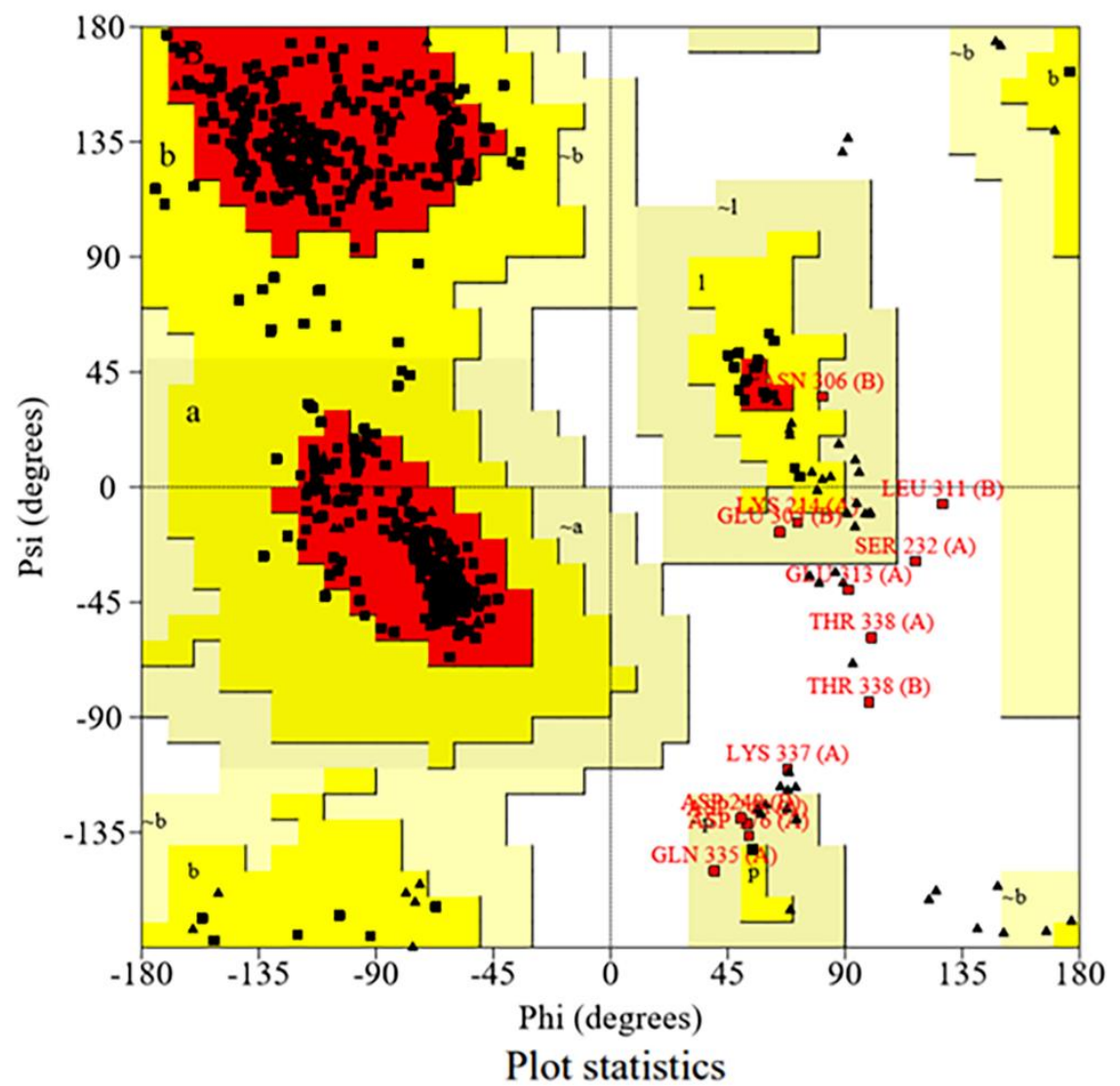

$\begin{array}{lrr}\text { Residues in most favoured regions [A,B, } \mathrm{L}] & 563 & 89.6 \% \\ \text { Residues in additional allowed regions [a,b,l,p] } & 52 & 8.3 \% \\ \text { Residues in generously allowed regions [ } \sim \mathrm{a}, \sim \mathrm{b}, \sim 1, \sim \mathrm{p}] & 7 & 1.1 \% \\ \text { Residues in disallowed regions } & 6 & 1.0 \% \\ & -\cdots & -\cdots-. \\ \text { Number of non-glycine and non-proline residues } & 628 & 100.0 \%\end{array}$

Figure 3. Ramachandran plot displaying structure validation of the ATPase domain of $C$. difficile GyrB protein.

\section{ADMET analysis}

In the process of drug development, many drug failures occur at early and late discovery pipeline because of poor ADME (Absorption, Distribution, Metabolism, and Excretion) and toxicity properties [48, 7375]. Therefore, it is important to address these issues in the early phase of drug discovery. Herein, the 139 flavonoids (known metabolites) were extracted from a total of 480 flavonoids from the PhytoHub database 
and were then considered for the ADMET analysis. Initially, Molinspiration program $[48,76]$ was employed to calculate various principal descriptors of ADME based on Lipinski rule of five for all molecules. Keeping the threshold value as 0 nviolations, the flavonoids were screened out. For instance, there were 62 out of 139 known metabolites violating the Ro5. Thus, 77 molecules were in comparable zone with 0 nviolations (Figure 4). Subsequently, these 77 molecules were subjected to oral toxicity analysis $[45,48,77,78]$. Moreover, the focused molecules were evaluated their toxicity based on the $\mathrm{LD}_{50}$ and Tox class values [45]. Out of 77 molecules, 35 compounds were found to be in criteria (listed in Table S1). Finally, these 35 known flavonoids metabolites were then subjected to toxicity parameter assessment (Figure 5, discussed later). Note that, there are several reported flavonoids which follow the Lipinski rule of five and possess the acceptable ADMET properties. Some of the widely known flavonoids have shown good ADMET properties with many biological properties such as anti-microbial, anti-cancer, anti-inflamatory, etc. as reported in previous studies [79-81]. Epicatechin and catechin, well known flavonoids, have shown good ADME properties with zero voilations and were found to exhibit anti-inflammatory against phospholipase A2, COX, LOX and anti-proliferative activity towards cervical, colon cancer respectively [82]. Gossypetin and Taxifolin, the known antiviral inhibitors, were found to be in range of Lipinski rule of five demonstrating their potential to be a drug like candidate [83]. This is another confirmation showing that flavonoids have good bioavailability with various biological activities. The 2D structures of all 35 flavonoids were represented in Figure S1.

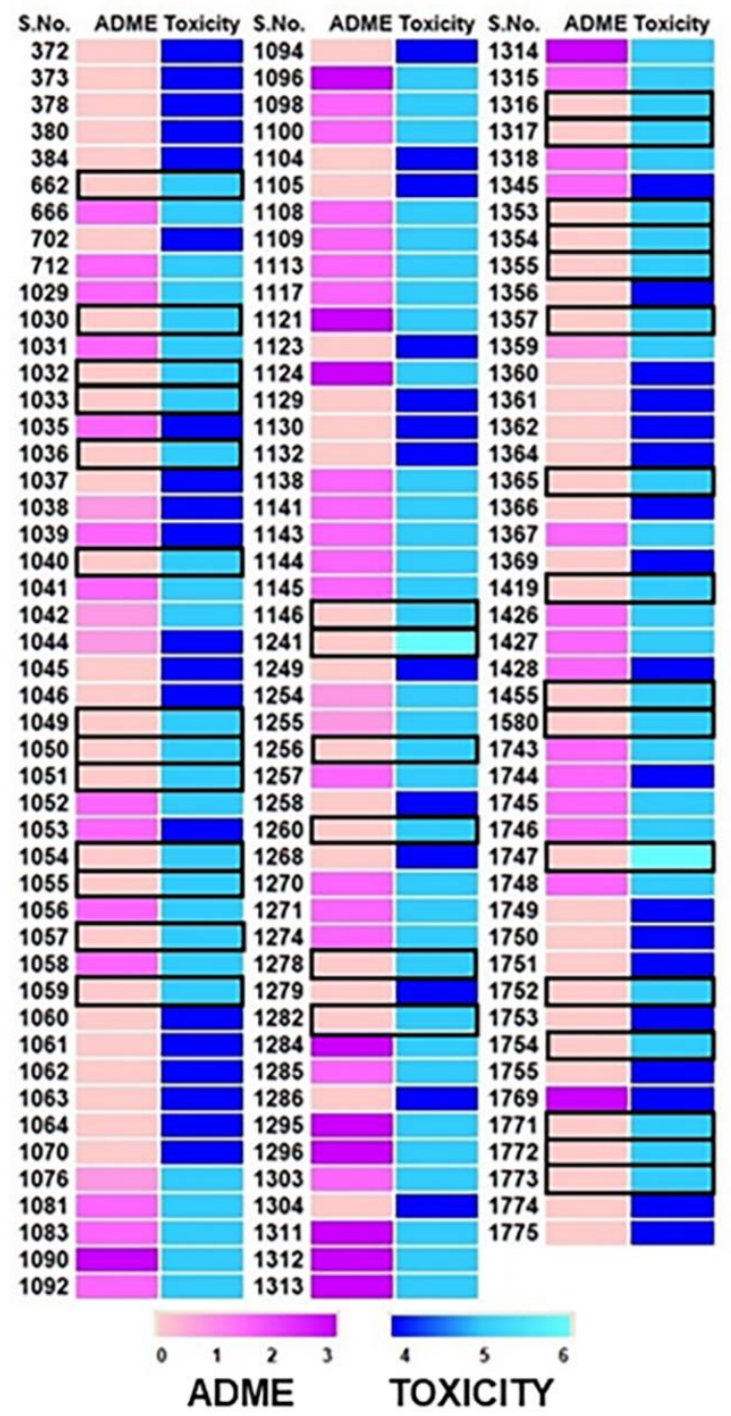

Figure 4. ADMET analysis of small molecules using Molinspiration and ProTox server.

\section{Toxicity assessment}

A total of 35 flavonoids which efficaciously passed the Lipinski Rule of Five and oral toxicity analysis was exposed for toxicity analysis. The flavonoids were screened out in comparison to the reference GyrB inhibitor novobiocin. The graphical representation for toxicity criteria was plotted using DataWarrior program (Figure 
5). The spheres in blue, red, and green colors indicate the high toxicity, low toxicity, and no toxicity, respectively. After calculations, only 25 flavonoids (i.e., 662, 1030, 1032, 1033, 1036, 1049, 1050, 1051, 1054, 1055, 1146, 1241, 1256, 1278, 1282, 1317, 1365, 1419, 1455, 1747, 1752, 1772, 1040, 1057 and 1059; Figure S1) were found to have no mutagenicity, tumorigenicity, irritant, and reproductive effect, in good agreement with the reported biogical activities [81, 82, 84-86]. Therefore, these molecules were then subjected to binding affinity prediction against the ATPase domain of GyrB using molecular docking analysis.

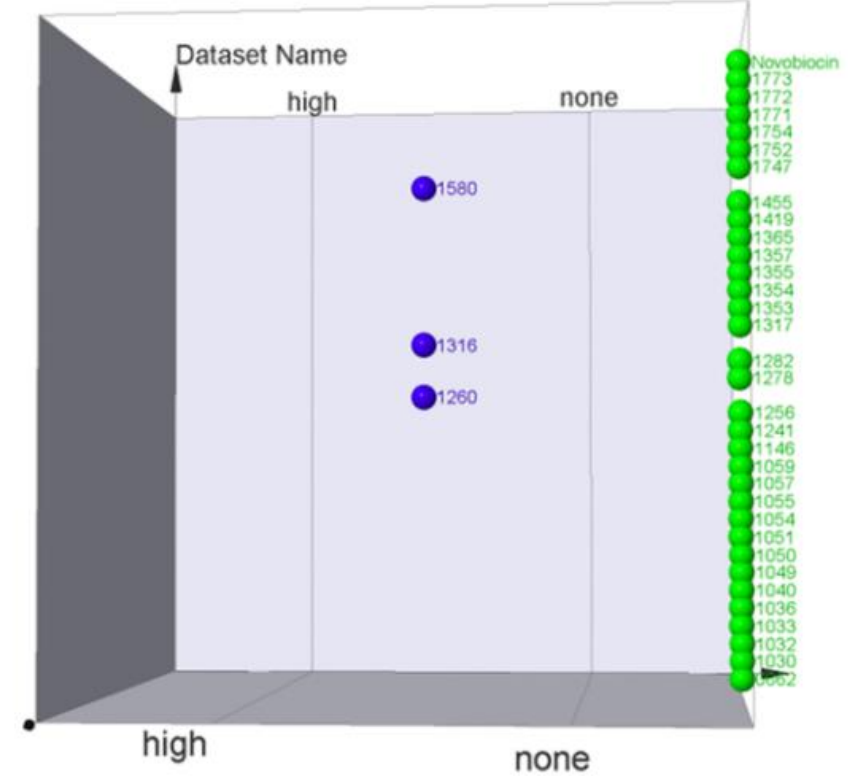

(a) Mutagenicity

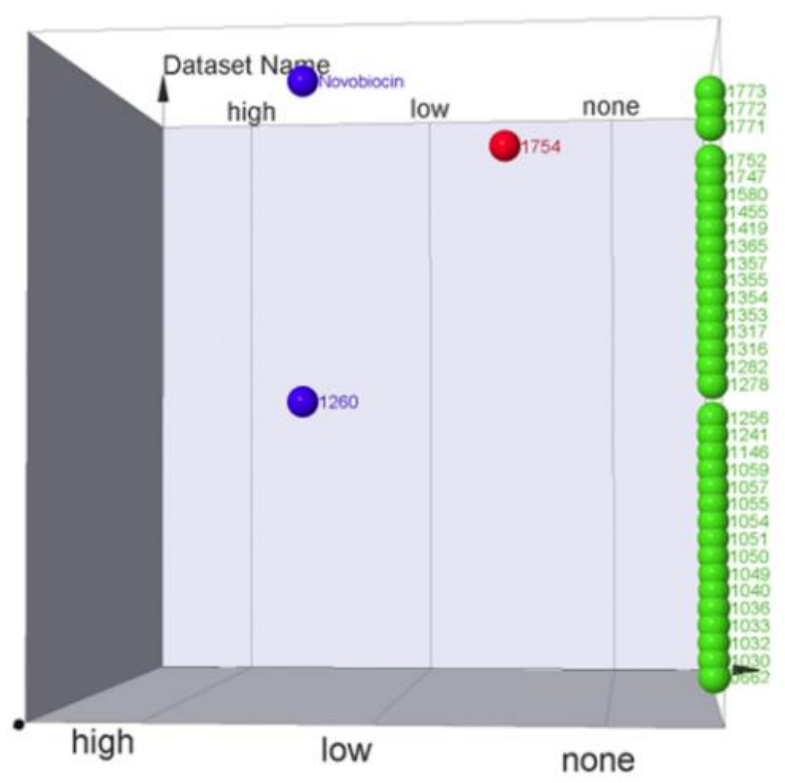

(c) Irritant

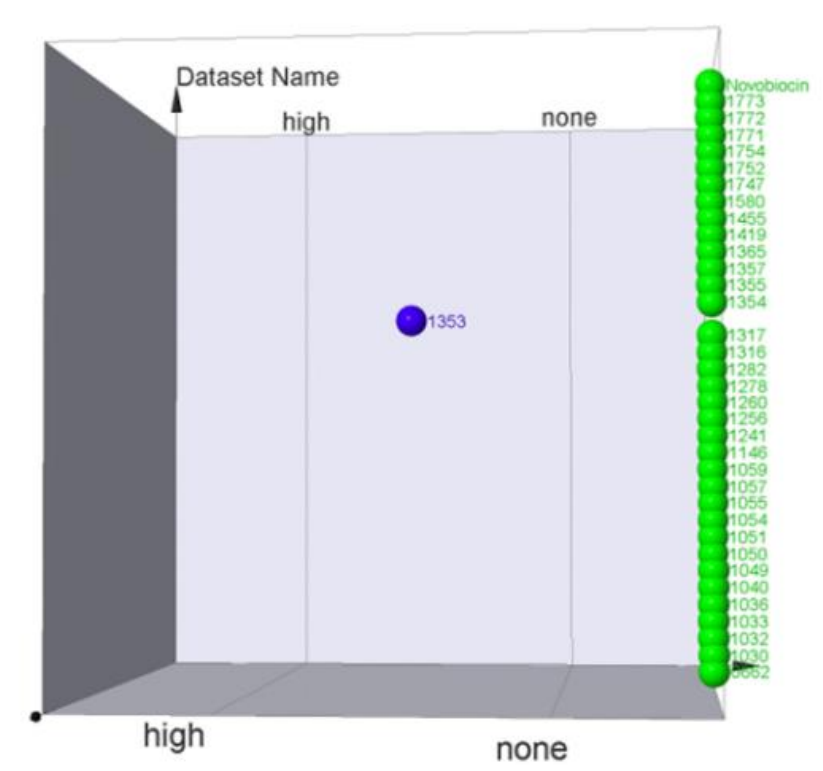

(b) Tumorigenicity

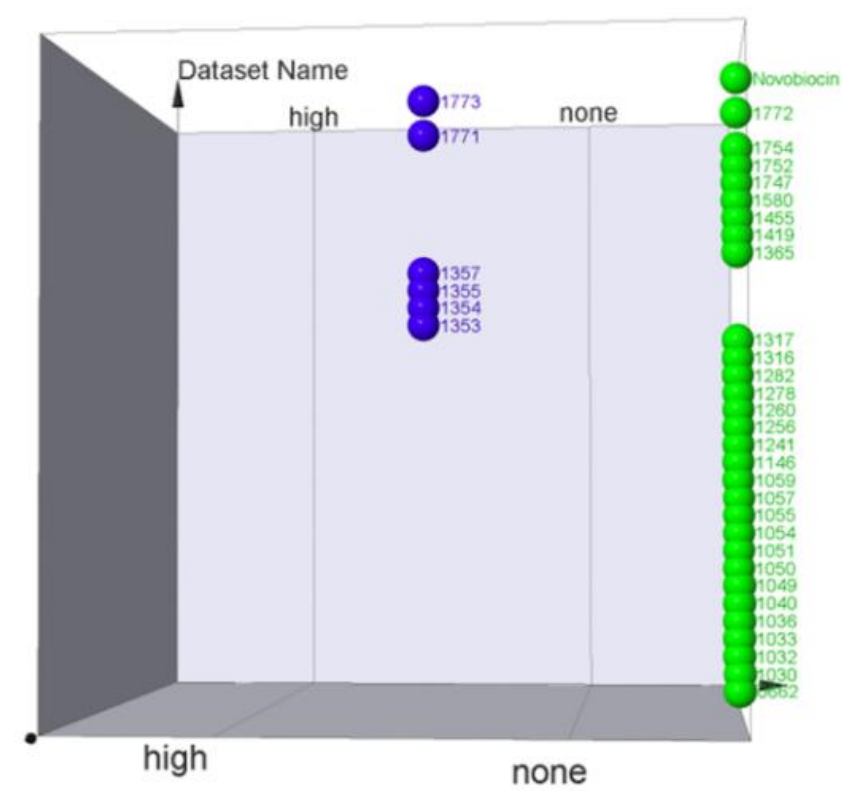

(d) Reproductive effect

Figure 5. Toxicity analysis for 35 known metabolites of flavonoids in terms of (a) mutagenicity (b) tumorigenicity (c) irritant and (d) reproductive effect. 


\section{Molecular docking}

To identify the most potent candidate for the treatment of CDI, the screened 25 molecules were docked into the ATPase domain of $C$. difficile GyrB using FlexX program. The docking score of each flavonoid is summarized in Figure 6 . The obtained results revealed that the reference GyrB inhibitor novobiocin showed the docking energy of $-19.00 \mathrm{kcal} / \mathrm{mol}$, and among 25 flavonoids, there were seventeen compounds that show good binding affinity in the range of the novobiocin (i.e., 662 (isorhamnetin; $-24.26 \mathrm{kcal} / \mathrm{mol}), 1033((-$ )-epicatechin 5-O-sulfate; -22.35 kcal/mol), 1036 ((-)-Epicatechin sulfate; -23.83 kcal/mol), 1050 (3'-OMethyl-(-)-epicatechin 5-O-sulfate; -23.47 kcal/mol), 1054 (4'-O-Methyl-(-)-epicatechin 5-O-sulfate; -23.19 $\mathrm{kcal} / \mathrm{mol}$ ), 1241 (epicatechin; -22.00 kcal/mol), 1241 (epicatechin;-22.00 kcal $/ \mathrm{mol}), 1256 \quad$ (4-(1Carboxyphenyl)beta-D-glucuronic acid; $-25.24 \mathrm{kcal} / \mathrm{mol}), 1278$ Isovanillic acid-3-O-sulfate; -20.36 kcal $/ \mathrm{mol}$ ), 1282 (methylvanillate;-12.86 kcal/mol), 1365 (calycosin; -20.89 kcal $/ \mathrm{mol}$ ), 1419 (petunidin; -19.52 kcal/mol), 1455 (malvidin; -20.78 kcal/mol), 1747 (3'-O-methyl-epicatechin; -20.89 kcal/mol), 1752 (4-hydroxybenzoic acid -3-sulfate; -18.17 kcal/mol), 1040 ((epi)catechin O-sulfate; -24.01 kcal/mol), 1057 (4'-Omethyl(epi)catechin O-sulfate; -22.23 kcal/mol) and 1059 (4'-O-Methyl(epi)gallocatechin O-sulfate; -20.76 $\mathrm{kcal} / \mathrm{mol})$. Interstingly, there were six flavonoids showing the significantly higher binding strength than the novobiocin, including 1030 ((-)-epicatechin 3'-O-sulfate), 1032 ((-)-epicatechin 4'-O-sulfate), 1049 (3'-OMethyl-(-)-epicatechin 4-O-sulfate), 1051 (3'-O-methyl-(-)-epicatechin 7-O-sulfate), 1055 (4'-O-Methyl-(-)epicatechin 7-O-sulfate) and 1317 (quercetin sulfate) with the docking energy of $-30.10,-29.42,-27.75$, $27.71,-28.79$ and $-26.41 \mathrm{kcal} / \mathrm{mol}$, respectively. Notably, the docked orientation of these flavonoids in the ATP-binding pocket of GyrB was somewhat similar to that of novobiocin (Figure 7). Altogether, these six flavonoids could likely be potent molecules for the treatment of CDI.

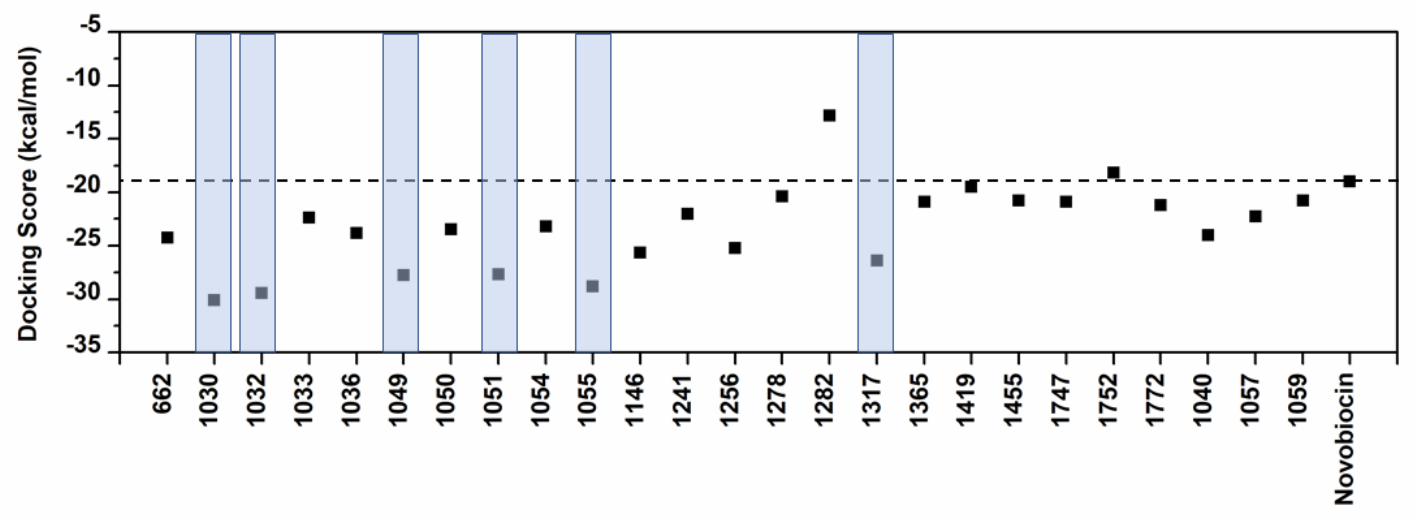

Figure 6. FlexX docking score for 25 flavonoids in complex with the ATPase domain of $C$. difficile GyrB protein. Note that the top six compounds with docking energy significantly lower than the novobiocin ((i.e., 1030 ((-)-epicatechin 3'-Osulfate), 1032 ((-)-epicatechin 4'-O-sulfate), 1049 (3'-O-Methyl-(-)-epicatechin 4-O-sulfate), 1051 (3'-O-methyl-(-)epicatechin 7-O-sulfate), 1055 (4'-O-Methyl-(-)-epicatechin 7-O-sulfate) and 1317 (quercetin sulfate)) are highlighted in blue boxes.
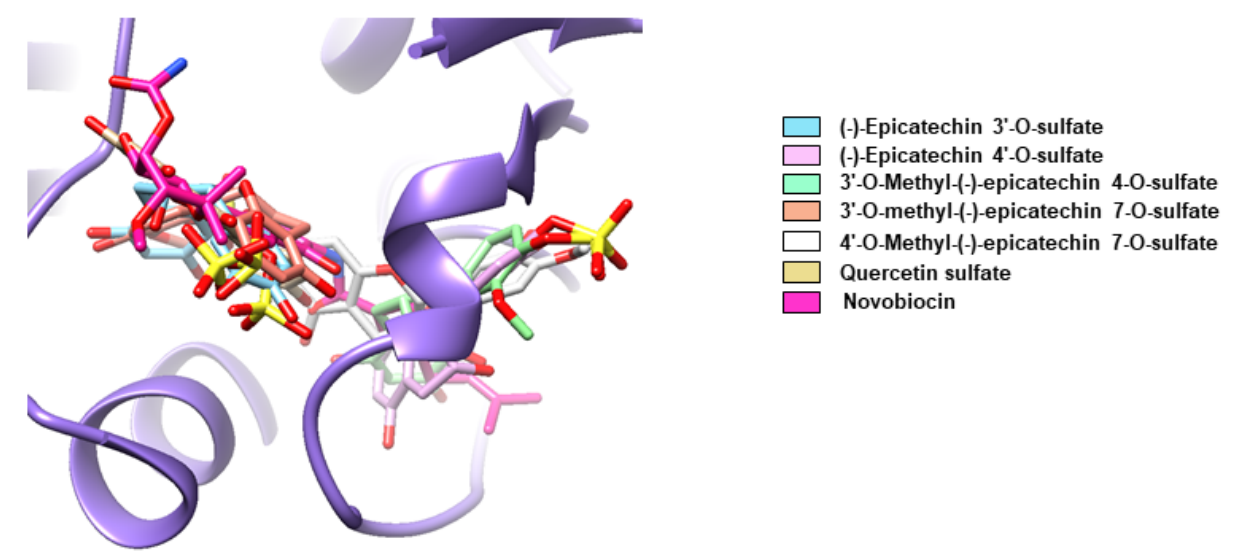

Figure 7. Superimposed structures of 1030 ((-)-epicatechin 3'-O-sulfate), 1032 ((-)-epicatechin 4'-O-sulfate), 1049 (3'-O-Methyl-(-)-epicatechin 4-O-sulfate), 1051 (3'-O-methyl-(-)-epicatechin 7-O-sulfate), 1055 (4'-O- 
Methyl-(-)-epicatechin 7-O-sulfate) and 1317 (quercetin sulfate) from the docking study with that of novobiocin in the ATP-binding pocket of GyrB.

The overall binding mode of top six compounds in the ATP-binding pocket is illustrated in Figure 8. The dashed line represents hydrogen bond $(\mathrm{H}$-bond), whereas green color label represents hydrophobic residues and spline segment highlights the hydrophobic contact. In all of the top compounds, F103 residue of GyrB was involved in the hydrophobic interaction. (-)-Epicatechin 3'-O-sulfate interacted with GyrB, forming $\mathrm{H}$ bonds with N45, E49, R75, G76, K102 and F103, whereas E49, R75, G76, I77, P78 and F103 were involved in hydrophobic interactions (Figure 8a). (-)-Epicatechin 4'-O-sulfate could form eight H-bonds with D98, H98, A99, K101, V117 and R22 from the N-terminal arm of the other monomer residues, whereas hydrophobic interactions were observed between residues D48, G100, F103, G104, K109, V117, R22 and the ligand (Figure 8b). 3'-O-Methyl-(-)-epicatechin 4'-O-sulfate was found to form eight $\mathrm{H}$-bonds with E41, N45, D48, H98, K101, R22 and hydrophobically interact with G100, F103, G104, G116 and V117 (Figure 8c). H-bonds formed between 3'-O-Methyl-(-)-epicatechin 7-O-sulfateand GyrB included residues R75, G76,K102, F103 and T164, while E49, R75, G76, I77, P78, F103 and K109 made hydrophobic contacts with this compound (Figure 8d). 4'-O-Methyl-(-)-epicatechin 7-O-sulfate formed seven $\mathrm{H}$-bonds with residues N45, D48, H98, A99, G116 and R22 and hydrophobically contacted with D48, H98, G100, F103, K109, G116, V117 and R22 (Figure 8e). The inteaction of Quercetin sulfate and GyrB involved seven H-bonds with residues N45, D48, R75, G76 and K109 and hydrophobic contacts with residues R75, P78, F103 and K109 (Figure 8f). Novobiocin interacted with GyrB, forming four H-bonds with residues Q9, R75, K109 and G116, while hydrophobic interactions with this compound was made by residues R75, P78, F103, K109, G116, V117 and R22 (Figure 8g). However, the the docked novobiocin orientation in complex with ATPase domain of $C$. difficile GyrB was different from the crystal structure of ATPase domain of T. thermophilus GyrB in complex with novobiocin [32] due to the differences between the two bacterial species.

It should be noted that the hydroxyl group $(-\mathrm{OH})$ group on the phenyl ring of the flavonoids were mainly involved in the $\mathrm{H}$-bond formations. Altogether, the interaction pattern of all the top six flavonoids shares some key binding residues with the reference novobiocin. This highlights the importance of the present study as it analyzes potent compounds that could efficiently replace novobiocin which could bind and occupy the same binding cavity in the ATPase domain of GyrB enzyme. However, further experimental validation should be conducted. 


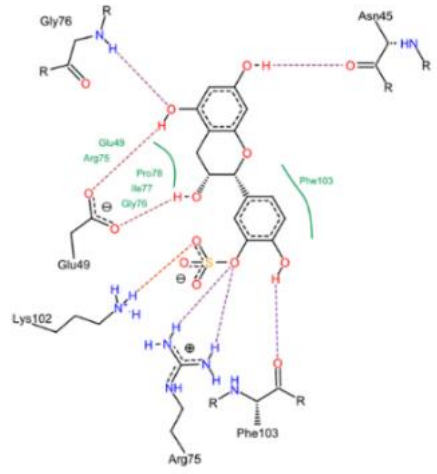

(a)

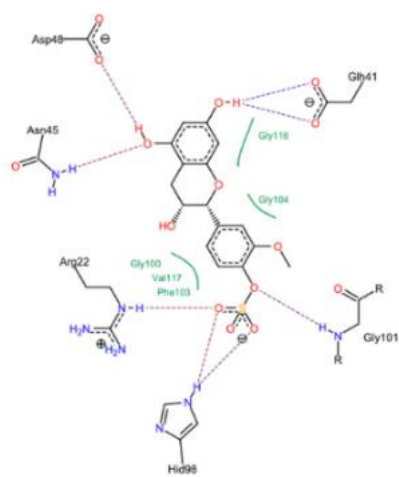

(c)

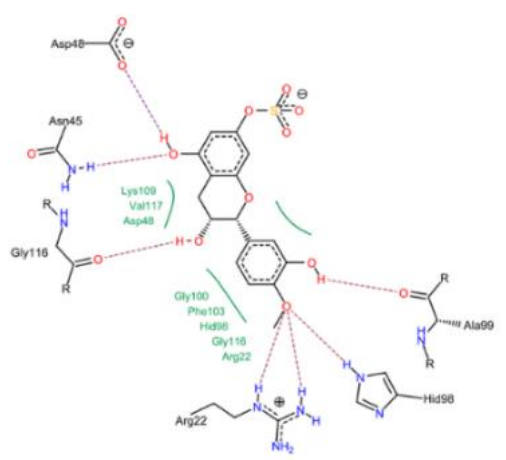

(e)

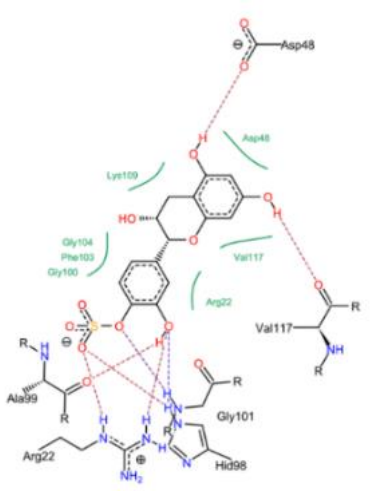

(b)

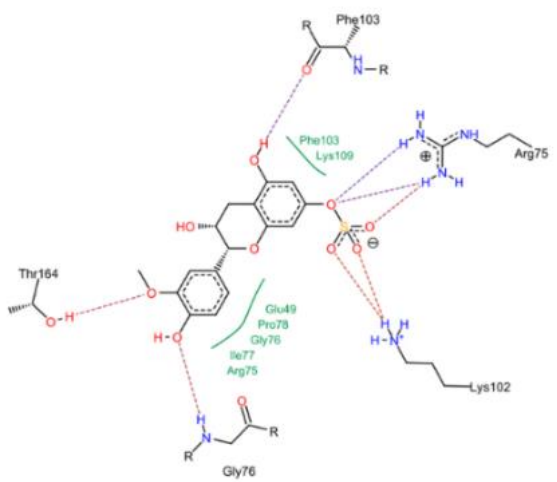

(d)

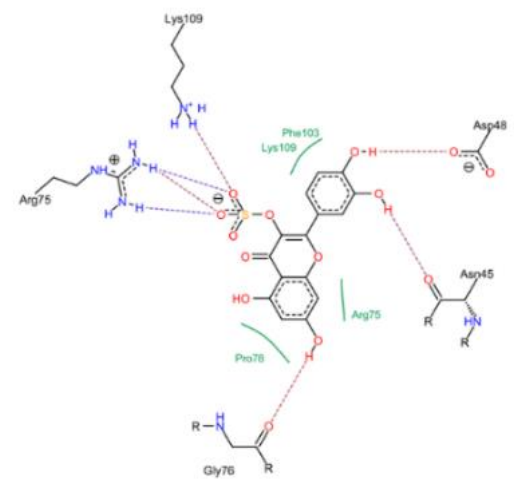

(f)

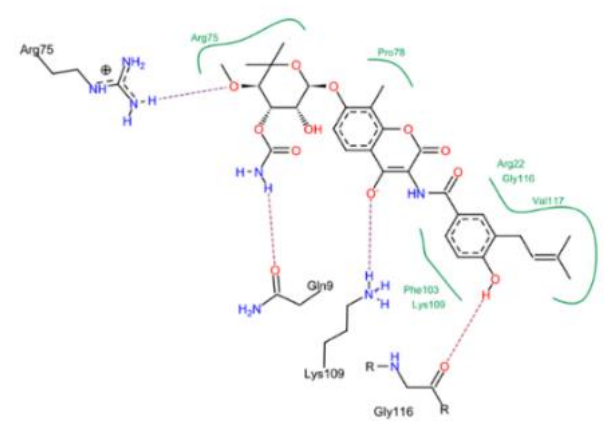

(g)

Figure 8. Interaction Diagram of protein-ligand complexes of top four compounds and reference molecule with GyrB protein (a) (-)-epicatechin 3'-O-sulfate, (b) (-)-epicatechin 4'-O-sulfate, (c) 3'-O-methyl-(-)epicatechin 4-O-sulfate, (d) 3'-O-methyl-(-)-epicatechin 7-O-sulfate, (e) 4'-O-Methyl-(-)-epicatechin 7-Osulfate, (f) quercetin sulfate, and (g) novobiocin. 


\section{System stability of simulated complexes}

To inspect the overall stability of the simulated protein-ligand complexes during the entire MD run, root mean square deviation (RMSD) of the protein backbone was calculated within the $5.0 \AA$ sphere of the ligand. As shown in Figure 9, RMSDs of almost all complexes were within the same range ( 1-2 $\AA$ ) except the GyrB1317 complex, which showed dramatic deviation up to approximately $3.0 \AA$ of RMSD value that may indicate the lower overall stability of this complex during the simulated time. However, RMSD of all complexes became more stable when the simulation time was increased and were very slightly deviated in the vicinity of the last 10 ns. Therefore, the MD trajectories from 40 to $50 \mathrm{~ns}$, which were considered reaching their equilibrated conformations were then extracted to explore protein-ligand binding affinity in terms of binding free energy $\left(\Delta \boldsymbol{G}_{\text {bind }}\right)$.

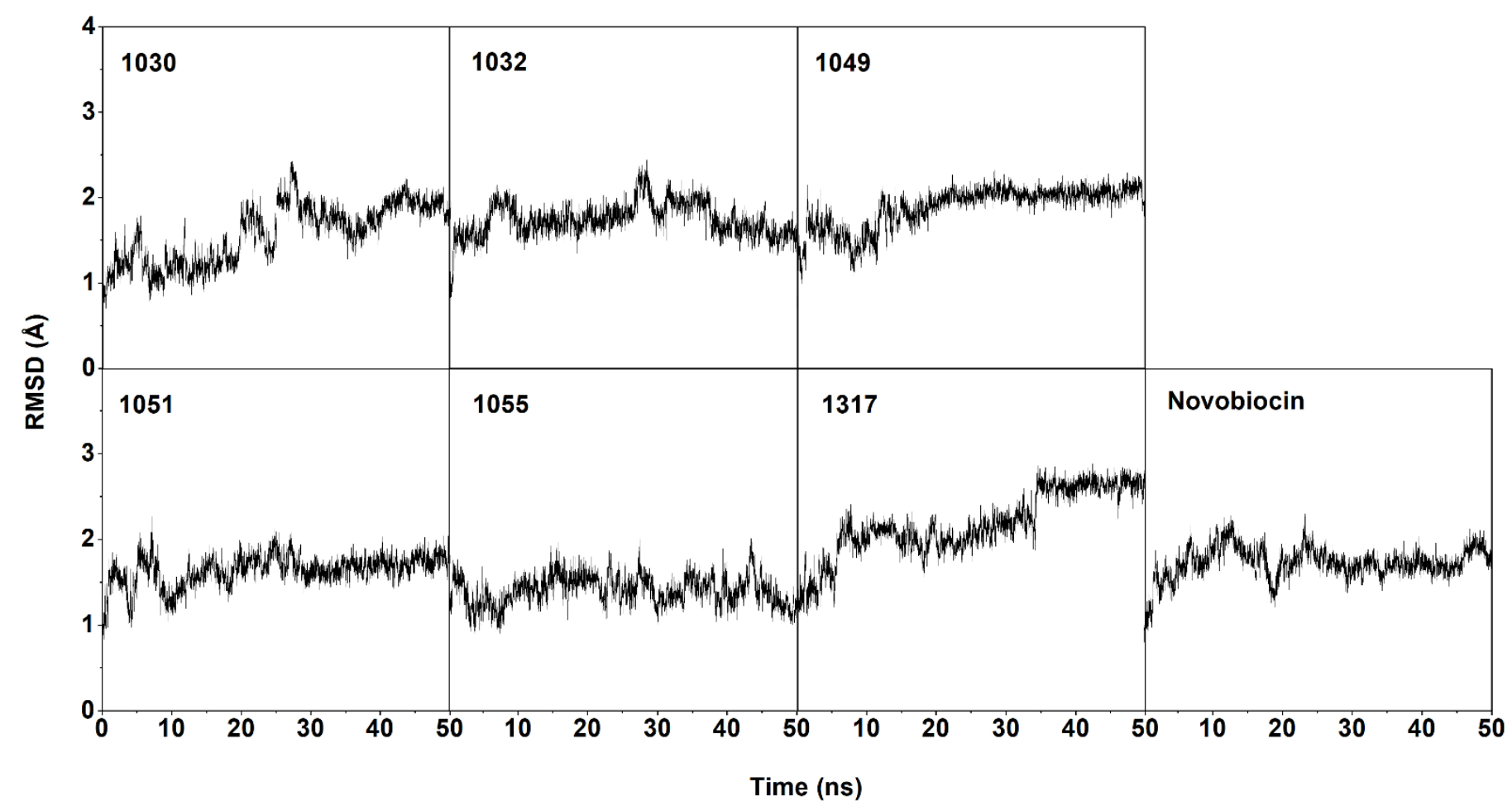

Figure 9. Backbone RMSD plot within the 5.0 Ă sphere of the ligand.

\section{Binding Free Energy}

Binding free energy $\left(\Delta G_{\text {bind }}\right)$ calculations sufficiently define the coupling strength between protein and ligand, and it is therefore known as a parameter for estimation of protein-ligand binding affinity in the process of drug discovery [87]. $\Delta G_{\text {bind }}$ calculation based on the solvated interaction energy (SIE) was applied to estimate the binding affinity of all GyrB-candidate complexes. The $\Delta G_{\text {bind }}$ values of the six screened flavonoids from the last $10 \mathrm{~ns}$ are listed in table 1 in comparison to the known inhibitor, novobiocin. According to the molecular mechanics $\left(\Delta E_{\mathrm{MM}}=\Delta \mathrm{E}_{\mathrm{vdW}}+\Delta \mathrm{E}_{\text {ele }}\right)$ calculations, we found that the electrostatic attraction is the main interactive energy contributing to the process of molecular complexation between GyrB and six candidate compounds. When the reaction field and cavity were taken into account, the $\Delta G_{\text {bind }}$ value of the studied complexes was obtained and used as the representative parameter to compare protein-ligand binding capability among candidate compounds. The $\Delta G_{\text {bind }}$ results showed that compound $1030\left(\Delta G_{\text {bind }}\right.$ of -8.45 $\mathrm{kcal} / \mathrm{mol})$ has higher binding affinity toward GyrB than the novobiocin $(-8.32 \mathrm{kcal} / \mathrm{mol})$, while the other compounds showed lower binding capability compared to novobiocin. Even though these candidate compounds exhibited lower $\Delta G_{\text {bind, }}$ we suggested that the compounds $1051\left(\Delta G_{\text {bind }}\right.$ of $\left.-7.50 \mathrm{kcal} / \mathrm{mol}\right), 1055$ $\left(\Delta G_{\text {bind }}\right.$ of $\left.-7.41 \mathrm{kcal} / \mathrm{mol}\right)$ and $1317\left(\Delta G_{\text {bind }}\right.$ of $\left.-7.16 \mathrm{kcal} / \mathrm{mol}\right)$ remained interesting as their calculated $\Delta G_{\text {bind }}$ value is lower than $-7 \mathrm{kcal} / \mathrm{mol}$ (considered as a second top among screened compounds). However, other two compounds including $1032\left(\Delta G_{\text {bind }}\right.$ of $\left.-6.59 \mathrm{kcal} / \mathrm{mol}\right)$ and $1049\left(\Delta G_{\text {bind }}\right.$ of $\left.-6.52 \mathrm{kcal} / \mathrm{mol}\right)$ revealed dramatically higher $\Delta G_{\text {bind }}$ than the novobiocin, indicating the lower level of binding capability towards GyrB and could be neglected to be explored further. To sum up, compound 1030 has been considered to plausibly exhibit the greatest binding affinity to GyrB while compounds 1032 and 1049 could be assumed to be least effective of binding among screened candidates. For other remaining candidate compounds (1051, 1055, 
and 1317), we assumed that their capability seems to be similar in magnitude based upon the predicted $\Delta G_{\text {bind. }}$. Additionally, we noted that the implications of number of surrounding atoms and SASA towards binding features of all studied complexes are in good agreement with the calculated $\Delta G_{\text {bind }}$ as discussed below.

Table 1. $\Delta G_{\text {bind }}$ values $(\mathrm{kcal} / \mathrm{mol})$ of candidate compounds as well as novobiocin in complex with GyrB calculated with the SIE method using alpha, gamma, and constant coefficients of $0.10,0.01$, and -2.89 , respectively.

\begin{tabular}{|c|c|c|c|c|c|}
\hline \multirow{2}{*}{ Candidate Compound } & \multicolumn{5}{|c|}{ Energy Components } \\
\hline & $\Delta \mathrm{E}_{\mathrm{vdW}}$ & $\Delta \mathrm{E}_{\text {ele }}$ & $\begin{array}{c}\text { reaction filed } \\
(\mathrm{RF})\end{array}$ & cavity & $\Delta G_{b i n d}$ \\
\hline 1030 & $-36.93 \pm 0.30$ & $-88.31 \pm 1.31$ & $79.86 \pm 0.89$ & $-7.72 \pm 0.03$ & $-8.45 \pm 0.05$ \\
\hline 1032 & $-32.61 \pm 0.28$ & $-44.38 \pm 0.79$ & $48.38 \pm 0.62$ & $-6.67 \pm 0.06$ & $-6.59 \pm 0.04$ \\
\hline 1049 & $-32.90 \pm 0.36$ & $-56.92 \pm 0.93$ & $63.37 \pm 0.82$ & $-8.24 \pm 0.04$ & $-6.52 \pm 0.05$ \\
\hline 1051 & $-40.20 \pm 0.29$ & $-35.01 \pm 0.66$ & $38.91 \pm 0.58$ & $-7.75 \pm 0.03$ & $-7.50 \pm 0.04$ \\
\hline 1055 & $-36.66 \pm 0.31$ & $-58.65 \pm 0.67$ & $60.05 \pm 0.66$ & $-7.90 \pm 0.08$ & $-7.41 \pm 0.06$ \\
\hline 1317 & $-32.18 \pm 0.27$ & $-50.51 \pm 0.57$ & $48.94 \pm 0.37$ & $-7.05 \pm 0.03$ & $-7.16 \pm 0.04$ \\
\hline Novobiocin & $-53.20 \pm 0.40$ & $-33.96 \pm 0.53$ & $46.48 \pm 0.43$ & $-11.04 \pm 0.08$ & $-8.31 \pm 0.06$ \\
\hline
\end{tabular}

\section{Number of surrounding atoms and solvent accessibility}

The non-covalent contacts of any atoms within the $5.0 \AA$ sphere of the ligand were identified by calculating the distance between each pair of atoms as depicted in Figure 10A. The number of surrounding atoms averaged in the last $10 \mathrm{~ns}$ of each candidate compound was counted and raked in the order of 1051 (278 atoms) > 1030 (246 atoms) > 1049 (230 atoms) 1055 (229 atoms) = 1317 (229 atoms) > 1032 (222 atoms). It could be estimated that compounds 1051 and 1030 tend to have greater intermolecular interactions, which are related to stronger protein-ligand recognition within the cleft of binding site, while compound 1032 may occur in least non-covalent interactions among candidate compounds. However, we found that; even though compound 1051 could have greater contacts with surrounding amino acid residues, the observed SASA in the binding pocket remained high (Figure10B), implying that these water molecules may somehow interrupt and reduce the interaction strength during GyrB-1051 complex formation. In addition, predicted number of surrounding atoms of compound 1317, which exhibits a low-SASA feature as discussed below has a lower level of contact than compound 1030.

Furthermore, the accessibility of the water acting as a solvent in the protein binding pocket was taken into consideration, since it could affect the ligand binding affinity. To measure the surface area occupied by water molecules, the calculation of solvent accessible surface area (SASA) on the amino acid residues within the $5.0 \AA$ sphere of the ligand was carried out and the results were illustrated in the Figure10B. It was observed that SASA value of all studied complex, at the beginning of simulated time was seen to be fluctuated ranging from slight to gradual deviation; however, SASA of all simulated complexes became more stable when they reached into their equilibrated state (40-50 ns). To compare the SASA feature of all candidate compounds, we set the average GyrB-novobiocin's SASA value in the last 10 ns of $820.82 \AA^{2}$ for a reference and we thus found that GyrB-1317 and GyrB-1030 showed a significant lower SASA value (avg. SASA of 619.04 , and $670.31 \AA^{2}$ ) than a reference complex, indicating well-fitted orientation/conformation within the binding pocket, which may exhibit a greater binding affinity than any other screened compounds. On the contrary, binding of compound 1032 and 1049 to GyrB binding site resulted in the huge accessibility of water molecules (avg. SASA of $840.66 \AA^{2}$, and 869.11 $\AA^{2}$ respectively) that could probably reduce the binding capability due to interfering the protein-ligand intermolecular interactions. Whereas, candidate compounds 1051 (avg. SASA of $771.24 \AA^{2}$ ) and 1055 (avg. SASA of $774.41 \AA^{2}$ ) have gradually lower value of SASA when compared to GyrB-novobiocin, implying affinity of these two compounds towards GyrB may be satisfactory. 
For the lines of evidence regarding $\Delta G_{\text {bind, }}$ number of surrounding atoms, and SASA, we suggested that compound 1030 could be the most superior in terms of binding affinity towards GyrB and could exhibit higher potency than the known inhibitor (novobiocin) while compounds 1051, 1055 and 1317 may have a potential to become specific GyrB inhibitors. These four flavonoid compounds identified from this study could become viable candidates for further investigation necessary for the development of therapeutic agents targeting GyrB.

(A)

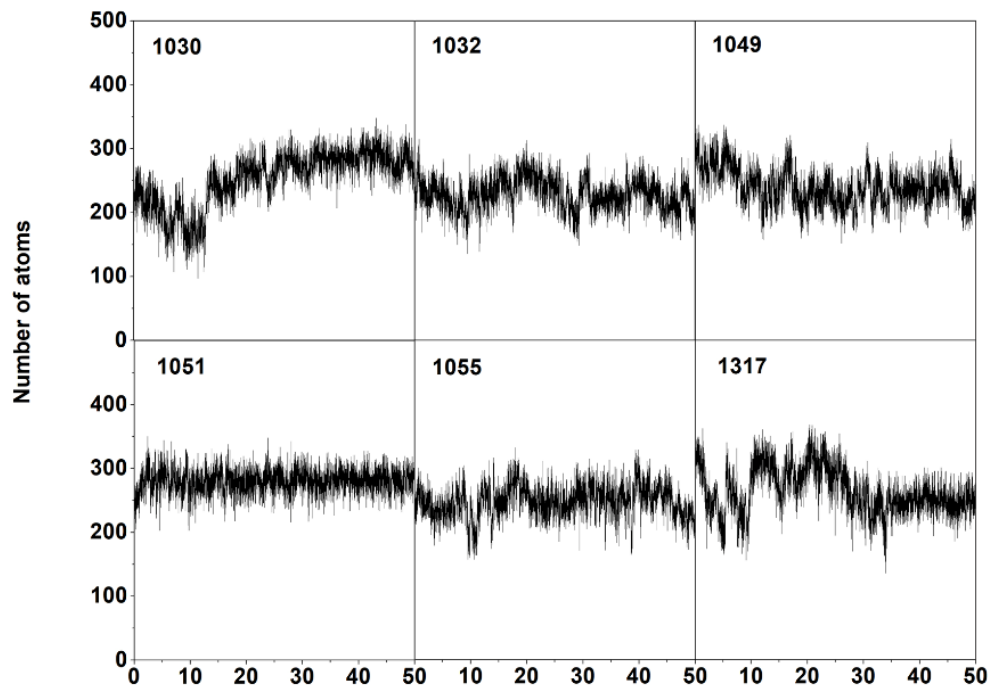

(B)

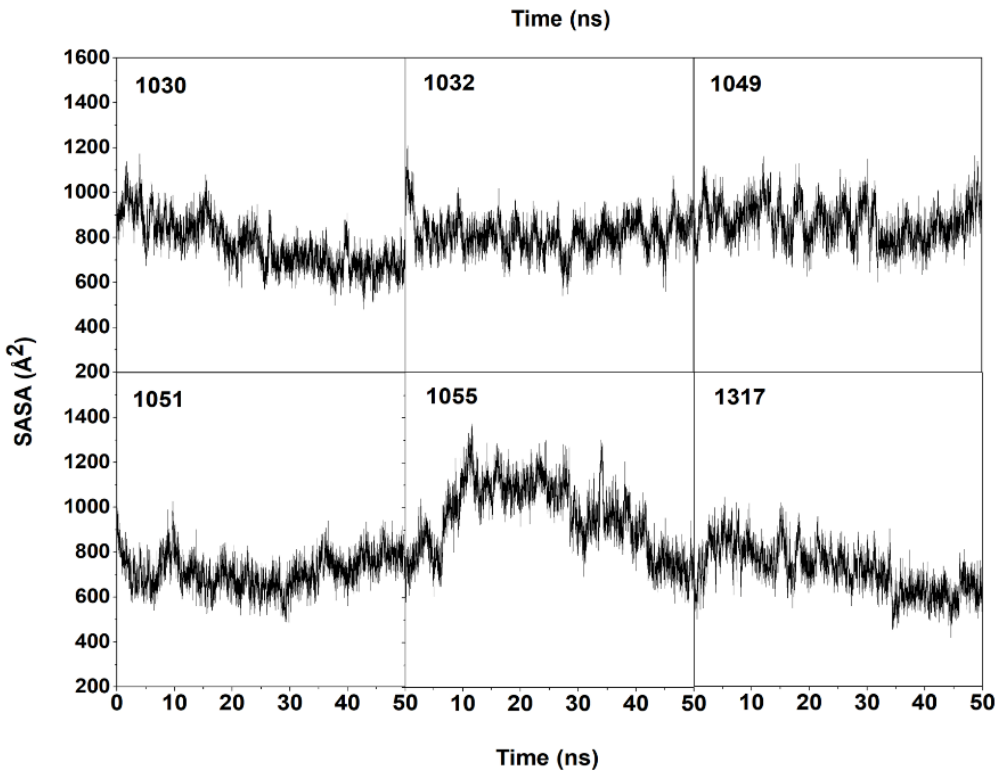

Figure 10. (A) Number of surrounding atoms and (B) SASA on the amino acid residues within the $5.0 \AA$ sphere of each candidate compound.

\section{CONCLUSION}

GyrB is highly essential for DNA replication process across all bacterial genera, making it an attractive drug target for the development of a novel antibacterial agent. Conceptually, there is an urgent need to identify such potent lead molecules as they will have lesser side effects compared to synthetic available drugs. Also, the pre-clinical investigation of the drugs for calculating their pharmacokinetic properties via in silico strategy is vital for its long-term success. The present study aimed to identify potential drug candidates against GyrB protein of $C$. difficile for the treatment of CDI. Initially, we screened out the compounds from PhytoHub database based on ADMET properties and toxicity parameters. Subsequently, the screened flavonoids were predicted for the binding affinity against GyrB using docking approach. The virtual screening results revealed that, among the 139 known metabolites, only six flavonoids, including (-)-epicatechin 3'-O-sulfate, (-)epicatechin 4'-O-sulfate, 3'-O-methyl-(-)-epicatechin 4-O-sulfate, 3'-O-methyl-(-)-epicatechin 7-O-sulfate, 4'O-methyl-(-)-epicatechin 7-O-sulfate, and quercetin sulfate have good bioavailability and no toxicity along with higher binding affinity than the reference novobiocin. Six potent molecules with higher binding affinity as 
compared to novobiocin were selected for simulation studies and free energy calculations. According to SASA and number of contact atom analysis, we found that compound 1030 ((-)-epicatechin 3'-O-sulfate) has less accessibility to water molecules and has good number of contact atoms which in turn lead to efficient binding of 1030 ((-)-epicatechin 3'-O-sulfate) to GyrB. Binding free energy calculations based on SIE method showed that 1030 has the highest binding efficiency with lower solvent accessibility at the ATP-binding pocket than the novobiocin. In this regard, we can propose that these flavonoids could likely be potent lead molecules for the treatment of CDI via targeting GyrB enzyme. This study thus had efficiently derived a potent bioactive compound (-)-epicatechin 3'-O-sulfate against GyrB for the CDI treatment. Therefore, we propose that flavonoids could possibly have inhibitory action towards GyrB and will help to target other antibacterial diseases too. Overall, this is the first report of flavonoids inhibitory action against GyrB for the management of CDI.

Acknowledgments: The authors would like to acknowledge the financial support from Center of Excellence on Medical Biotechnology (CEMB), The S\&T Postgraduate Education and Research Development Office (PERDO), The Commission on Higher Education (CHE), Thailand. K.V. would also like to thank Ratchadapisek Somphot Fund for postdoctoral fellowship. We also thank Biocatalyst and Environmental Biotechnology Research Unit, Faculty of Science, $\mathrm{CU}$, for facility and computing resources. P.Mai. thanks, Chulalongkorn University of a short-term visit grant.

Conflicts of Interest: The authors declare no conflict of interest.

\section{REFERENCES}

1. Ghose C. Clostridium difficile infection in the twenty-first century. Emerg Microbes Infect. 2013;2(9):e62.

2. Khanna S, Pardi DS, Aronson SL, Kammer PP, Orenstein R, St Sauver JL, et al. The epidemiology of communityacquired Clostridium difficile infection: a population-based study. Am J Gastroenterol. 2012;107(1):89-95.

3. Nagy E. What do we know about the diagnostics, treatment and epidemiology of Clostridioides (Clostridium) difficile infection in Europe? J Infect Chemother. 2018;24(3):164-70.

4. Rajendram M, Hurley KA, Foss MH, Thornton KM, Moore JT, Shaw JT, et al. Gyramides prevent bacterial growth by inhibiting DNA gyrase and altering chromosome topology. ACS Chem Biol. 2014;9(6):1312-9.

5. Wang JC. Cellular roles of DNA topoisomerases: a molecular perspective. Nat Rev Mol Cell Biol. 2002;3(6):43040.

6. Costenaro L, Grossmann JG, Ebel C, Maxwell A. Modular structure of the full-length DNA gyrase B subunit revealed by small-angle X-ray scattering. Structure. 2007;15(3):329-39.

7. Stokes SS, Vemula R, Pucci MJ. Advancement of GyrB Inhibitors for Treatment of Infections Caused by Mycobacterium tuberculosis and Non-tuberculous Mycobacteria. ACS Infect Dis. 2020;6(6):1323-31.

8. Stanger FV, Dehio C, Schirmer T. Structure of the N-terminal Gyrase B fragment in complex with ADP. Pi reveals rigid-body motion induced by ATP hydrolysis. PLoS One. 2014;9(9).

9. Singh SB. Discovery and development of kibdelomycin, a new class of broad-spectrum antibiotics targeting the clinically proven bacterial type II topoisomerase. Bioorg Med Chem. 2016;24(24):6291-7.

10. Mathur T, Barman TK, Kumar M, Singh D, Kumar R, Khera MK, et al. In Vitro and In Vivo Activities of DS-2969b, a Novel GyrB Inhibitor, against Clostridium difficile. Antimicrob Agents Chemother. 2018;62(4):e02157-17.

11. Durcik M, Tomašič T, Zidar N, Zega A, Kikelj D, Mašič LP, et al. ATP-competitive DNA gyrase and topoisomerase IV inhibitors as antibacterial agents. Expert Opin Ther Pat. 2019;29(3):171-80.

12. Bryskier A. Antibiotics and antibacterial agents: classifications and structure-activity relationship. Antimicrobial Agents: ASM; 2005. p. 13-38.

13. Heide L. Genetic engineering of antibiotic biosynthesis for the generation of new aminocoumarins. Biotechnol Adv. 2009;27(6):1006-14.

14. Azam MA, Thathan J, Jubie S. Dual targeting DNA gyrase B (GyrB) and topoisomerse IV (ParE) inhibitors: a review. Bioorg Chem. 2015;62:41-63.

15. Collin F, Karkare S, Maxwell A. Exploiting bacterial DNA gyrase as a drug target: current state and perspectives. Appl Microbiol Biotechnol. 2011;92(3):479-97.

16. Bisacchi GS, Manchester JI. A new-class antibacterial $\square$ almost. Lessons in drug discovery and development: A critical analysis of more than 50 years of effort toward ATPase inhibitors of DNA gyrase and topoisomerase IV. ACS infect Dis. 2015;1(1):4-41.

17. Fedorowicz J, Sączewski J. Modifications of quinolones and fluoroquinolones: hybrid compounds and dual-action molecules. Monatsh Chem. 2018;149(7):1199-245.

18. Kumar S, Pandey AK. Chemistry and Biological Activities of Flavonoids: An Overview. Sci World J. 2013;2013:16.

19. Galati G, O'brien PJ. Potential toxicity of flavonoids and other dietary phenolics: significance for their chemopreventive and anticancer properties. Free Radical Biol Med. 2004;37(3):287-303.

20. Fang Y, Lu Y, Zang X, Wu T, Qi X, Pan S, et al. 3D-QSAR and docking studies of flavonoids as potent Escherichia coli inhibitors. Sci Rep. 2016;6:23634.

21. Plaper A, Golob M, Hafner I, Oblak M, Šolmajer T, Jerala R. Characterization of quercetin binding site on DNA gyrase. Biochem Biophys Res Commun. 2003;306(2):530-6. 
22. Bhardwaj VK, Purohit R. A new insight into protein-protein interactions and the effect of conformational alterations in PCNA. Int J Biol Macromol. 2020;148:999-1009.

23. Rajendran V, Sethumadhavan R. Drug resistance mechanism of PncA in Mycobacterium tuberculosis. J Biomol Struct Dyn. 2014;32(2):209-21.

24. Rajendran V, Gopalakrishnan C, Purohit R. Impact of point mutation P29S in RAC1 on tumorigenesis. Tumor Biol. 2016;37(11):15293-304.

25. Bhardwaj V, Purohit R. Computational investigation on effect of mutations in PCNA resulting in structural perturbations and inhibition of mismatch repair pathway. J Biomol Struct Dyn. 2020;38(7):1963-74.

26. Bhardwaj VK, Purohit R. Targeting the protein-protein interface pocket of Aurora-A-TPX2 complex: Rational drug design and validation. J Biomol Struct Dyn. 2020:1-17.

27. Bhardwaj VK, Singh R, Sharma J, Rajendran V, Purohit R, Kumar S. Identification of bioactive molecules from Tea plant as SARS-CoV-2 main protease inhibitors. J Biomol Struct Dyn. 2020:1-13.

28. S Tsutsumi L, B Owusu Y, G Hurdle J, Sun D. Progress in the discovery of treatments for C. difficile infection: a clinical and medicinal chemistry review. Curr Top Med Chem. 2014;14(1):152-75.

29. Britton RA, Young VB. Role of the intestinal microbiota in resistance to colonization by Clostridium difficile. Gastroenterology. 2014;146(6):1547-53.

30. Jarrad AM, Karoli T, Blaskovich MA, Lyras D, Cooper MA. Clostridium difficile drug pipeline: challenges in discovery and development of new agents. J Med Chem. 2015;58(13):5164-85.

31. Bento da Silva A, Giacomoni F, Pavot B, Fillatre Y, Rothwell J, Bartolomé Sualdea B, et al., editors. PhytoHub V1.4: A new release for the online database dedicated to food phytochemicals and their human metabolites. The 1 International Conference on Food Bioactives \& Health; 2016 2016-09-13; Norwich, United Kingdom.

32. Lamour V, Hoermann L, Jeltsch J-M, Oudet P, Moras D. An Open Conformation of the Thermus thermophilusGyrase B ATP-binding Domain. J Biol Chem. 2002;277(21):18947-53.

33. Bordoli L, Kiefer F, Arnold K, Benkert P, Battey J, Schwede T. Protein structure homology modeling using SWISSMODEL workspace. Nat Protoc. 2009;4(1):1-13.

34. Guex N, Peitsch MC. SWISS-MODEL and the Swiss-Pdb Viewer: an environment for comparative protein modeling. Electrophoresis. 1997;18(15):2714-23.

35. Schwede T, Kopp J, Guex N, Peitsch MC. SWISS-MODEL: an automated protein homology-modeling server. Nucleic Acids Res. 2003;31(13):3381-5.

36. Lovell SC, Davis IW, Arendall WB, 3rd, de Bakker PI, Word JM, Prisant MG, et al. Structure validation by Calpha geometry: phi,psi and Cbeta deviation. Proteins. 2003;50(3):437-50.

37. Olsson MHM, Søndergaard CR, Rostkowski M, Jensen JH. PROPKA3: Consistent Treatment of Internal and Surface Residues in Empirical pKa Predictions. J Chem Theory Comput. 2011;7(2):525-37.

38. Maier JA, Martinez C, Kasavajhala K, Wickstrom L, Hauser KE, Simmerling C. ff14SB: Improving the Accuracy of Protein Side Chain and Backbone Parameters from ff99SB. J Chem Theory Comput. 2015;11(8):3696-713.

39. Shoichet BK. Virtual screening of chemical libraries. Nature. 2004;432(7019):862-5.

40. Karthick V, Ramanathan K. Virtual screening for oseltamivir-resistant a (H5N1) influenza neuraminidase from traditional Chinese medicine database: a combined molecular docking with molecular dynamics approach. SpringerPlus. 2013;2(1):115.

41. Bielska E, Lucas X, Czerwoniec A, M. Kasprzak J, H. Kaminska K, M. Bujnicki J. REVIEW PAPER<BR>Virtual screening strategies in drug design - methods and applications. BioTechnologia. 2014;92(3):249-64.

42. Kitchen DB, Decornez H, Furr JR, Bajorath J. Docking and scoring in virtual screening for drug discovery: methods and applications. Nat Rev Drug Discov. 2004;3(11):935-49.

43. Chackalamannil S, Rotella D, Ward S. Comprehensive Medicinal Chemistry III: Elsevier; 2017.

44. Lipinski CA, Lombardo F, Dominy BW, Feeney PJ. Experimental and computational approaches to estimate solubility and permeability in drug discovery and development settings1PII of original article: S0169409X(96)00423-1. The article was originally published in Advanced Drug Delivery Reviews 23 (1997) 3-25.1. Adv Drug Del Rev. 2001;46(1):3-26.

45. Drwal MN, Banerjee P, Dunkel M, Wettig MR, Preissner R. ProTox: a web server for the in silico prediction of rodent oral toxicity. Nucleic Acids Res. 2014;42(Web Server issue):W53-8.

46. Meraj K, Mahto MK, Christina NB, Desai N, Shahbazi S, Bhaskar M. Molecular modeling, docking and ADMET studies towards development of novel Disopyramide analogs for potential inhibition of human voltage gated sodium channel proteins. Bioinformation. 2012;8(23):1139-46.

47. Ntie-Kang F. An in silico evaluation of the ADMET profile of the StreptomeDB database. Springerplus. 2013;2:353.

48. Verma K, Kannan K. Exploring $\beta$-Tubulin Inhibitors from Plant Origin using Computational Approach. Phytochem Anal. 2017;28(3):230-41.

49. Dixon SL, Jurs PC. Estimation of pKa for organic oxyacids using calculated atomic charges. J Comput Chem. 1993;14(12):1460-7.

50. Csizmadia F, Tsantili-Kakoulidou A, Panderi I, Darvas F. Prediction of Distribution Coefficient from Structure. 1. Estimation Method. J Pharm Sci. 1997;86(7):865-71.

51. Csizmadia P, editor MarvinSketch and MarvinView: molecule applets for the World Wide Web. Proceedings of ECSOC-3, the third international electronic conference on synthetic organic chemistry, September 1ą30; 1999. 
52. Petukh M, Stefl S, Alexov E. The role of protonation states in ligand-receptor recognition and binding. Curr Pharm Des. 2013;19(23):4182-90.

53. Onufriev AV, Alexov E. Protonation and pK changes in protein-ligand binding. Q Rev Biophys. 2013;46(2):181-209.

54. Frisch MJ, Trucks GW, Schlegel HB, Scuseria GE, Robb MA, Cheeseman JR, et al. Gaussian 16 Rev. A.03. Wallingford, CT2016.

55. Mahalapbutr P, Darai N, Panman W, Opasmahakul A, Kungwan N, Hannongbua S, et al. Atomistic mechanisms underlying the activation of the $G$ protein-coupled sweet receptor heterodimer by sugar alcohol recognition. Sci Rep. 2019;9(1):10205.

56. Mahalapbutr P, Thitinanthavet K, Kedkham T, Nguyen H, Dokmaisrijan S, Huynh L, et al. A theoretical study on the molecular encapsulation of luteolin and pinocembrin with various derivatized beta-cyclodextrins. J Mol Struct. 2019;1180:480-90.

57. Kramer B, Rarey M, Lengauer T. Evaluation of the FLEXX incremental construction algorithm for protein-ligand docking. Proteins: Structure, Function, and Bioinformatics. 1999;37(2):228-41.

58. Schellhammer I, Rarey MJPS, Function,, Bioinformatics. FlexX-Scan: Fast, structure-based virtual screening. 2004;57(3):504-17.

59. Wang J, Wolf RM, Caldwell JW, Kollman PA, Case DA. Development and testing of a general amber force field. J Comput Chem. 2005;26(1):114.

60. Jorgensen WL, Chandrasekhar J, Madura JD, Impey RW, Klein ML. Comparison of simple potential functions for simulating liquid water. J Chem Phys. 1983;79(2):926-35.

61. Kammarabutr J, Mahalapbutr P, Nutho B, Kungwan N, Rungrotmongkol T. Low susceptibility of asunaprevir towards R155K and D168A point mutations in HCV NS3/4A protease: A molecular dynamics simulation. J Mol Graph Model. 2019;89:122-30.

62. Kammarabutr J, Mahalapbutr P, Nutho B, Kungwan N, Rungrotmongkol T. Low susceptibility of asunaprevir towards R155K and D168A point mutations in HCV NS3/4A protease: A molecular dynamics simulation. J Mol Graphics Model. 2019;89:122-30.

63. Meeprasert A, Hannongbua S, Rungrotmongkol T. Key binding and susceptibility of NS3/4A serine protease inhibitors against hepatitis C virus. J Chem Inf Model. 2014;54(4):1208-17.

64. Nutho B, Mahalapbutr P, Hengphasatporn K, Pattaranggoon NC, Simanon N, Shigeta Y, et al. Why Are Lopinavir and Ritonavir Effective against the Newly Emerged Coronavirus 2019? Atomistic Insights into the Inhibitory Mechanisms. Biochemistry. 2020;59(18):1769-79.

65. Nutho B, Rungrotmongkol T. Binding recognition of substrates in NS2B/NS3 serine protease of Zika virus revealed by molecular dynamics simulations. J Mol Graph Model. 2019;92:227-35.

66. Darden T, York D, Pedersen L. Particle mesh Ewald: An N. $\log (\mathrm{N})$ method for Ewald sums in large systems. J Chem Phys. 1993;98(12):10089-92.

67. Ryckaert J-P, Ciccotti G, Berendsen HJ. Numerical integration of the cartesian equations of motion of a system with constraints: molecular dynamics of n-alkanes. J Comput Phys. 1977;23(3):327-41.

68. Uberuaga BP, Anghel M, Voter AFJTJocp. Synchronization of trajectories in canonical molecular-dynamics simulations: Observation, explanation, and exploitation. 2004;120(14):6363-74.

69. Berendsen HJ, Postma Jv, van Gunsteren WF, DiNola A, Haak JR. Molecular dynamics with coupling to an external bath. J Chem Phys. 1984;81(8):3684-90.

70. Naïm M, Bhat S, Rankin KN, Dennis S, Chowdhury SF, Siddiqi I, et al. Solvated Interaction Energy (SIE) for Scoring Protein-Ligand Binding Affinities. 1. Exploring the Parameter Space. J Chem Inf Model. 2007;47(1):122-33.

71. Roe DR, Cheatham TE, 3rd. PTRAJ and CPPTRAJ: Software for Processing and Analysis of Molecular Dynamics Trajectory Data. J Chem Theory Comput. 2013;9(7):3084-95.

72. Miller III BR, McGee Jr TD, Swails JM, Homeyer N, Gohlke H, Roitberg AE. MMPBSA. py: an efficient program for end-state free energy calculations. J Chem Theory Comput. 2012;8(9):3314-21.

73. Laskowski R, MacArthur M, Thornton J. PROCHECK: validation of protein-structure coordinates. 2006.

74. Dong J, Wang N-N, Yao Z-J, Zhang L, Cheng Y, Ouyang D, et al. ADMETlab: a platform for systematic ADMET evaluation based on a comprehensively collected ADMET database. J Cheminform. 2018;10(1):29.

75. Wang J, Urban L, Bojanic D. Maximising use of in vitro ADMET tools to predict in vivo bioavailability and safety. Expert Opin Drug Metab Toxicol. 2007;3(5):641-65.

76. Thangarasu P, Selvi ST, Manikandan A. Unveiling novel 2-cyclopropyl-3-ethynyl-4-(4-fluorophenyl) quinolines as GPCR ligands via PI3-kinase/PAR-1 antagonism and platelet aggregation valuations; development of a new class of anticancer drugs with thrombolytic effects. Bioorg Chem. 2018;81:468-80.

77. Lei T, Li Y, Song Y, Li D, Sun H, Hou T. ADMET evaluation in drug discovery: 15. Accurate prediction of rat oral acute toxicity using relevance vector machine and consensus modeling. J Cheminform. 2016;8(1):6.

78. Wang Y, Xing J, Xu Y, Zhou N, Peng J, Xiong Z, et al. In silico ADME/T modelling for rational drug design. Q Rev Biophys. 2015;48(4):488-515.

79. Yugandhar P, Kumar KK, Neeraja P, Savithramma N. Isolation, characterization and in silico docking studies of synergistic estrogen receptor a anticancer polyphenols from Syzygium alternifolium (Wt.) Walp. J Intercult Ethnopharmacol. 2017;6(3):296-310. 
80. Babu TMC, Rajesh SS, Bhaskar BV, Devi S, Rammohan A, Sivaraman T, et al. Molecular docking, molecular dynamics simulation, biological evaluation and 2D QSAR analysis of flavonoids from Syzygium alternifolium as potent anti-Helicobacter pylori agents. RSC advances. 2017;7(30):18277-92.

81. Iheagwam FN, Ogunlana OO, Ogunlana OE, Isewon I, Oyelade J. Potential anti-cancer flavonoids isolated from Caesalpinia bonduc young twigs and leaves: molecular docking and in silico studies. Bioinform Biol Insights. 2019;13:1177932218821371.

82. Yadavalli R, Peasari JR, Mamindla P, Mounika S, Ganugapati J. Phytochemical screening and in silico studies of flavonoids from Chlorella pyrenoidosa. Inform Med Unlocked. 2018;10:89-99.

83. Raj U, Varadwaj PK. Flavonoids as multi-target inhibitors for proteins associated with Ebola virus: In silico discovery using virtual screening and molecular docking studies. INTERDISCIP SCI. 2016;8(2):132-41.

84. Iftikhar $\mathrm{H}$, Rashid $\mathrm{S}$. Molecular docking studies of flavonoids for their inhibition pattern against $\beta$-catenin and pharmacophore model generation from experimentally known flavonoids to fabricate more potent inhibitors for Wnt signaling pathway. Pharmacogn Mag. 2014;10(Suppl 2):S264.

85. Fan Z-F, Ho S-T, Wen R, Fu Y, Zhang L, Wang J, et al. Design, Synthesis and Molecular Docking Analysis of Flavonoid Derivatives as Potential Telomerase Inhibitors. Molecules. 2019;24(17):3180.

86. Ortuno A, Benavente-Garcia O, Castillo J, Alcaraz M, Vicente V, Del Rio J. Beneficial action of Citrus flavonoids on multiple cancer-related biological pathways. Curr Cancer Drug Targets. 2007;7(8):795-809.

87. Bhardwaj VK, Singh R, Sharma J, Das P, Purohit R. Structural based study to identify new potential inhibitors for dual specificity tyrosine-phosphorylation- regulated kinase. Comput Methods Programs Biomed. 2020;194:105494.

2021 by the authors. Submitted for possible open access publication under the terms and conditions of the Creative Commons Attribution (CC BY NC) license (https://creativecommons.org/licenses/by-nc/4.0/). 